\title{
Electrospray Mass Spectrometric Characterization of Bimetallic Group 8 - Gold Clusters
}

\author{
Rajiv Bhalla, PaulJ Dyson* and Frank Lauterwasser \\ Department of Chemistry, The Univerity of York, Heslington, York, YO1O 5DD, UK \\ and J Scott McIndoe \\ Department of Chemistry, The University of Cambridge, Lensfield Road, Cambridge, CB2 IEW, UK
}

Received: 29 March 2000

Bimetallic clusters containing gold in conjunction with Group 8 metals have been intensively studied as it is proposed that these compounds may find uses as catalysts or materials (or as precursors to catalysts/materials). These types of compounds are very difficult to characterize by the mass spectrometric techniques traditionally used to analyse transition metal clusters. In this paper we report a derivatization method employing electrospray ionization mass spectrometry that is reliable and informative for these types of compounds.

Transition metal carbonyl clusters are essentially small metal aggregates containing typically between three and 50 metal atoms. Smaller than colloids, they are soluble in common organic solvents, and in addition possess well defined structures (thousands of examples having been characterized by X-ray crystallography) (1). Many clusters are active catalysts and operate under homogeneous conditions, or by heterogenizing the cluster, by attachment to supports such as silica and alumina surfaces and intrazeolite anchoring (2). Catalysis by clusters differs from that of mononuclear homogeneous catalysts in that multicentre bond activation may take place that can give rise to different products unavailable by other homogeneous methods (3).

A large number of bimetallic clusters have been prepared and characterized in the laboratory and some prominent review articles have been published (4), including one on those containing gold (5). There are also some impressive catalytic applications, too many to be listed here, but some examples include the use of $\mathrm{Ru}-\mathrm{Pt}$ clusters for alkyne hydrogenation (6), Rh-Co clusters for hydrosilyation (7), Fe-Ru clusters for isomerization of alkenes (8), and Pt-Au clusters for $\mathrm{H}_{2}$ $\mathrm{D}_{2}$ equilibration (9). It is not unreasonable to assume that many bimetallic compounds may have better homogeneous catalytic properties than homonuclear species since many alloys are superior to single component heterogeneous catalysts.

Despite the intense research activity in this area, characterization of the clusters relies extensively on single crystal X-ray diffraction methods, as spectroscopic techniques such as IR and NMR are of little help, and mass spectrometry tends to be quite unreliable. Good mass spectra of bimetallic clusters are difficult to obtain as they have, typically, low volatilities, high molecular weights, complicated isotopic distributions and competing fragmentation pathways.

\section{SYNTHETIC METHODOLOGY}

The synthesis of bimetallic gold/Group 8 metal clusters involves a metathesis reaction (usually referred to as an ionic coupling reaction by cluster chemists) between a gold(I) phosphine fragment, $\left[\mathrm{Au}\left(\mathrm{PR}_{3}\right)\right]^{+}$, and a cluster anion (10). The gold cation is generated in situ from the reaction of the corresponding chloride with a suitable chloride abstractor such as a silver or thallium salt. These reactions typically proceed in yields exceeding $80 \%$.

The $\left[\mathrm{Au}\left(\mathrm{PR}_{3}\right)\right]^{+}$fragment is isolobal to the hydride 
and, in principle, it is possible to replace any hydride in a cluster with the gold-phosphine fragment (although direct substitution of a hydride by a gold-phosphine fragment is usually not possible) (11). Clearly, steric effects play a part as the gold-phosphine unit is considerably more bulky than a hydride, and the nature of the phosphine ligand attached to the $\mathrm{Au}(\mathrm{I})$ ion also has a marked influence on the structure of the product. For example, dianionic clusters that react with two equivalents of the gold reagent can give rise to skeletal isomers. In certain cases the gold ions will cap opposite faces of the cluster whereas chelating phosphine-gold fragments such as $\left[\mathrm{AuPPh}_{2} \mathrm{CH}_{2} \mathrm{CH}_{2} \mathrm{PPh}_{2} \mathrm{Au}\right]^{2+}$ force the gold centres to cap the same face (10).

\section{MASS SPECTROMETRY}

Mass spectrometric characterization of clusters has improved dramatically over the last few years (12). The main developments have come from applying techniques such as laser desorption ionization (LDI) and electrospray ionization (ESI), which were developed with polymers and biomolecules in mind, to cluster molecules. Both LDI and ESI work very well for charged clusters (13) but both are less straightforward for the characterization of neutral clusters. With neutral clusters, LDI gives rise to extensive clustering, leading to extremely complicated spectra (14), and ESI requires the use of reagents to chemically induce charge onto the cluster (15). The use of ESI mass spectrometry in organometallic chemistry (including that of clusters) continues to expand, and we have now found that it is the only technique that gives reliable spectra of heteronuclear gold/Group 8 metal clusters.

The basic principle of electrospray ionization (ESI) involves spraying a solution of a substance through a capillary into a chamber, through which a stream of dry gas passes in the opposite direction to the spray (16). A potential of several $\mathrm{kV}$ is applied between the capillary and the chamber wall. Charged droplets are produced that become smaller as the solvent is evaporated, and eventually bare ions are formed which pass through a glass capillary into the pre-analyser region, where the remaining bath gas and residual solvent is pumped away. The ions are then focused through the lensing system into the analyser of the mass spectrometer.

A number of methods have been developed for the analysis of neutral metal carbonyl clusters using ESIMS (15). Because the oxygen of the carbonyl ligand is not appreciably basic, it will not protonate and hence species of the type $[\mathrm{M}+\mathrm{H}]^{+}$are not observed. Various other charged species have, however, been shown to derivatize neutral carbonyl clusters.

Silver (I) ions also behave as ionization agents with a limited number of neutral metal carbonyl clusters providing $[\mathrm{M}+\mathrm{Ag}]^{+}$and/or $[\mathrm{M}+\mathrm{Ag}+\text { solvent }]^{+}$type ions (depending on the solvent and the cone voltage) (17). It seems likely that the interaction is between an electron-rich metal-metal bond of a cluster and the silver ion to form a $\mathrm{M}_{2} \mathrm{Ag}$ triangle. Clusters of this type are known which are sufficiently stable to be characterised structurally, though all the examples have strongly electron donating ligands.

The alkali metal ions $\mathrm{Li}^{+}, \mathrm{Na}^{+}$and $\mathrm{K}^{+}$will also associate with very electron-rich clusters such as $\mathrm{Ru}_{3}(\mathrm{CO})_{9}\left(\mathrm{PPh}_{3}\right)_{3}$ to give species of the type $[\mathrm{M}+$ cation $]^{+}$. It is believed that the attachment of the ion is likely to be via an iso-carbonyl link of the type $\mathrm{L}_{\mathrm{n}} \mathrm{M}-\mathrm{C} \equiv \mathrm{O} \ldots \mathrm{Na}^{+}$.

The most effective and general method to date is the use of alkoxide ion as an ionization agent (15). The sample is dissolved in the corresponding alcohol, and a small quantity of sodium alkoxide solution is added. Nucleophilic attack by the alkoxide ion on a coordinated carbonyl ligand provides a charged species:

$$
\mathrm{L}_{\mathrm{n}} \mathrm{M}-\mathrm{CO}+\mathrm{OR}^{-} \rightarrow\left[\mathrm{L}_{\mathrm{n}} \mathrm{M}-\mathrm{CO}_{2} \mathrm{R}\right]^{-}
$$

The reaction represented by Equation (1) is quite general for metal carbonyl compounds, and is rapid and large equilibrium constants favour the product, all of which are ideal characteristics for a derivatization process. It is this alkoxide ion derivatization method that affords reliable and reproducible spectra of bimetallic gold/Group 8 metal clusters. Figure 1 shows the ESI-MS of the new cluster $\mathrm{Ru}_{6} \mathrm{C}(\mathrm{CO})_{16}\left\{\mathrm{AuPPh}_{2}\left(\mathrm{C}_{16} \mathrm{H}_{15}\right)\right\}_{2}{ }^{*}$ obtained from a methanolic solution containing sodium methoxide. Two peaks are observed, one of high intensity centred at $\mathrm{m} / \mathrm{z} 2276.67$ and one of lower intensity centred at $\mathrm{m} / \mathrm{z}$ 1655.57. Expansions of these two peaks are shown in Figure 2 together with calculated models for

\footnotetext{
* Preparation of $\mathrm{Ru}_{6} \mathrm{C}(\mathrm{CO})_{16}\left\{\mathrm{AuPPh}_{2}\left(\mathrm{C}_{16} \mathrm{H}_{15}\right)\right\}_{2}:$ The cluster $\left[\mathrm{Ru}_{6} \mathrm{C}(\mathrm{CO})_{16}\right][\mathrm{PPN}]_{2}(100 \mathrm{mg}, 0.047 \mathrm{mmol})$ (PPN $=$ the bis(triphenylphosphoranylidene)ammonium cation, $\mathrm{ClAuPPh}\left(\mathrm{C}_{16} \mathrm{H}_{15}\right)$ $(58 \mathrm{mg}, 0.093 \mathrm{mmol})\left(\mathrm{PPh}_{2}\left(\mathrm{C}_{16} \mathrm{H}_{15}=\right.\right.$ diphenyl[2.2] paracyclophanylphosphine) and $\mathrm{AgBF}_{4}(27 \mathrm{mg}, 0.0141 \mathrm{mmol})$ were dissolved in $\mathrm{CH}_{2} \mathrm{Cl}_{2}$ and stirred for $18 \mathrm{~h}$ at room temperature. The reaction mixture was filtered and the solvent removed in vacuo. The product was then recrys tallized from $\mathrm{CH}_{2} \mathrm{Cl}_{2}$-hexane mixture (yield $36 \mathrm{mg}$ ).
} 


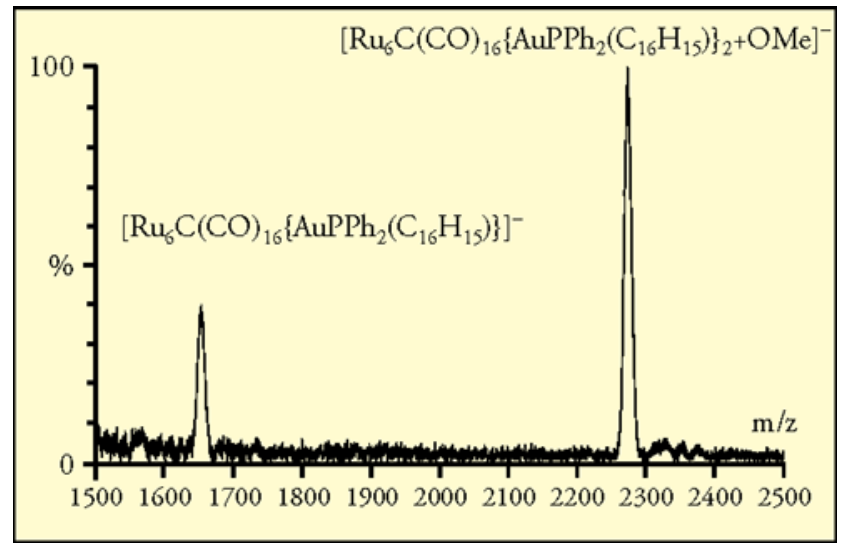

Figure 1 The ESI-MS of the new cluster

$\left[R u_{6} C(C O)_{16}\left\{A u P P h_{2}\left(C_{16} H_{15}\right)\right\}_{2}\right]$ obtained from a methanolic solution containing sodium methoxide, showing the $\left[R u_{6} C(C O)_{16} A u P P h_{2}\left(C_{16} H_{15}\right)\right]$ impurity.

comparison. The peak centred at $\mathrm{m} / \mathrm{z} 2276.67$ corresponds extremely well to intact cluster with a methoxide ion and the peak centred at $\mathrm{m} / \mathrm{z} 1655.6$ corresponds to the cluster anion $\left.\mathrm{Ru}_{6}(\mathrm{CO})_{16}\left\{\mathrm{AuPPh}_{2}\left(\mathrm{C}_{16} \mathrm{H}_{15}\right)\right\}\right]^{-}$which has not been methoxylated (see Figure 3). $\left.\mathrm{Ru}_{6}(\mathrm{CO})_{16}\left\{\mathrm{AuPPh}_{2}\left(\mathrm{C}_{16} \mathrm{H}_{15}\right)\right\}\right]^{-}$could not be identified by other spectroscopic methods or other mass spectrometric techniques as these give complicated fragmentation patterns that obscure the presence of the anion. The great advantage of derivatization ESI-MS is the fact that intact cluster species are detected due to the softness of the method.

We have investigated a range of related clusters using the same method and in each case found the results to be consistent with the compound reported above (some also containing the anion impurity).

\section{CONCLUSIONS AND OUTLOOK}

The potential role of bimetallic clusters containing gold with Group 8 metals in catalysis and materials is enormous. Reliable characterization of these compounds is not trivial and we have demonstrated a new method that gives reproducible molecular weight. data.

\section{ACKNOWLEDGEMENTS}

We would like to thank DMD Chiral Ltd for financial support (RB), the Royal Society for a University

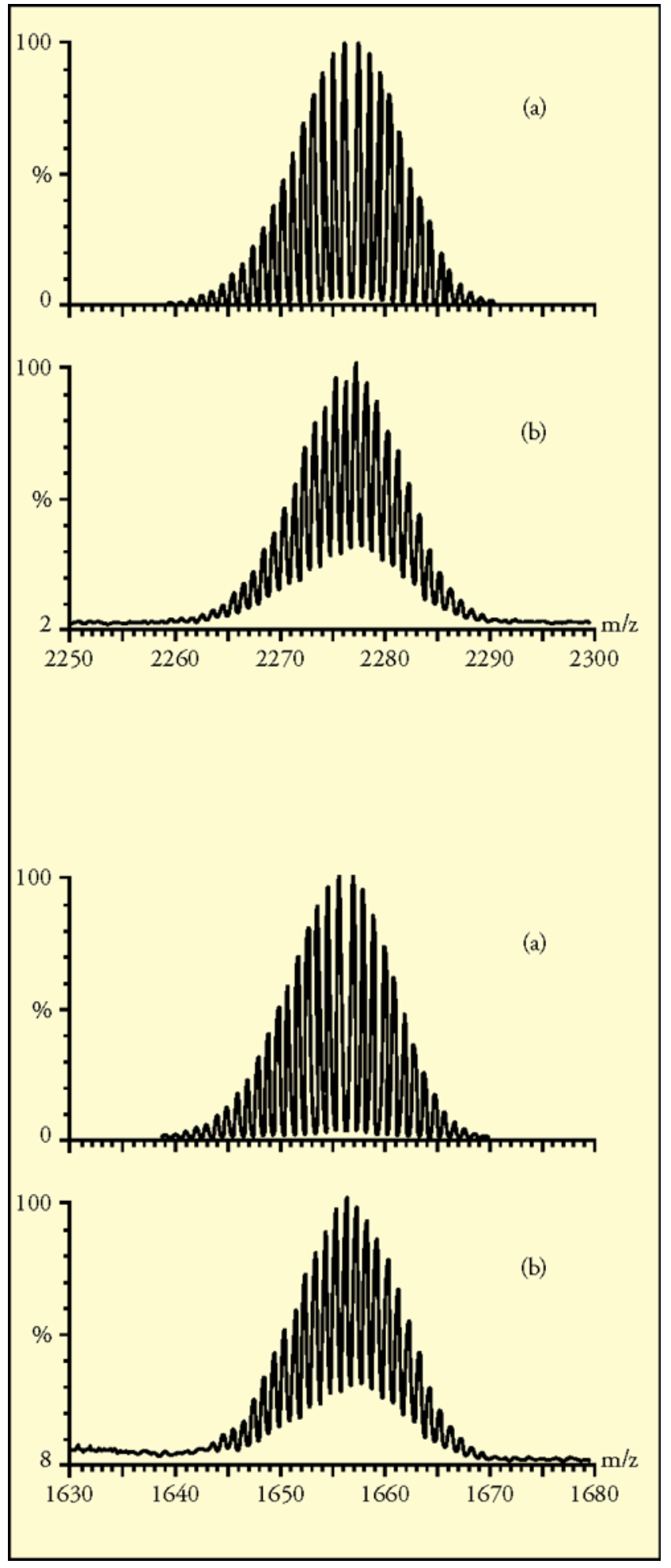

Figure 2 Comparison of (top) the peak envelope corresponding to $\left.\left[\mathrm{Ru}_{6} \mathrm{C}(\mathrm{CO})_{16} \mathrm{AuPPh}_{2}\left(\mathrm{C}_{16} \mathrm{H}_{15}\right)\right\}_{2}\right]$ and (bottom) the peak envelope corresponding to $\left[R u_{6} C(C O)_{16} A u P P b_{2}\left(C_{16} H_{15}\right)\right]$. (a) is the calculated model and $(b)$ is the observed spectrum. 


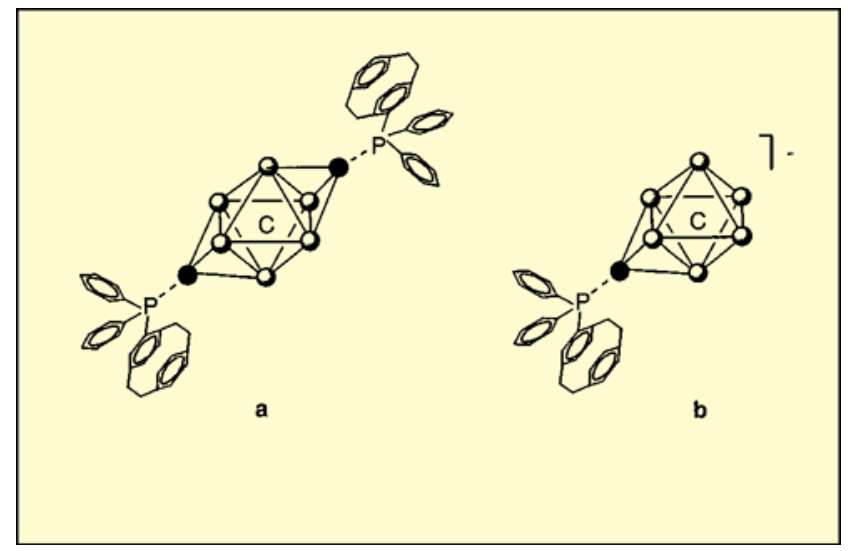

Figure 3 The proposed structures of (a) $\left[R u_{6} C(C O)_{16}\left[A u P P h_{2}\left(C_{16} H_{15}\right)\right\}_{2}\right]$ and (b) $\left[R u_{6} C(C O)_{16} \mathrm{AuPPh}_{2}\left(\mathrm{C}_{16} \mathrm{H}_{15}\right)\right]^{-}$: CO ligands have been omitted for clarity.

Research Fellowship (PJD), the ERASMUS scheme for assistance (FL) and the New Zealand Foundation for Science and Technology for a Postdoctoral Fellowship (JSM, contract CAM801).

\section{ABOUT THE AUTHORS}

Dr Dyson commenced his research career under the supervision of Professor Brian Johnson at The University of Cambridge working on the synthesis and characterization of ruthenium clusters. He moved with his supervisor to The University of Edinburgh where he completed his PhD. He then spent four years as a lecturer and Royal Society University Research Fellow at Imperial College, moving to The University of York in 1998. He also runs his own company, DMD Chiral Ltd, that is actively involved in the design and manufacture of chiral phosphine co-catalysts. Dr Bhalla obtained his $\mathrm{PhD}$ from the University of Manchester, moving to the University of East Anglia for a brief spell before moving to the University of York in 1997. Mr Lauterwasser is a student at Aachen University and spent a three month period in Dr Dyson's research group at the University of York. Dr McIndoe obtained his $\mathrm{PhD}$ at The University of
Waikato in New Zealand. Since 1998 he has been based at the University of Cambridge and works on a joint project spanning the Universities of Cambridge, Edinburgh and York.

\section{REFERENCES}

1 There are numerous books as well as an entire journal (Journal of Cluster Science) devoted to the subject of clusters. For example, see: (a) 'Transition Metal Clusters' ed. B.EG. Johnson, John Wiley \& Sons, Chichester, 1980; (b) 'Metal-Metal Bonds and Clusters in Chemistry and Catalysis', Ed. J.P. Fackler, Jr., Plenum, New York, 1990; (c) 'Introduction to Cluster Chemistry', D.M.P. Mingos and D.J. Wales, Prentice-Hall, London, 1990; (d) 'The Synergy Between Dynamics and Reactivity at Clusters and Surfaces', Ed. L.J. Farrugia, Kluwer, Dordrecht, 1995

2 'Catalysis by Di- and Polynuclear Metal Cluster Complexes', eds. R.D. Adams and F.A. Cotton, Wiley-VCH, New York, 1998

3 (a) N. Chatani, Y. Ishii, Y. le, F. Kakituchi and S. Murai, J. Org. Chem, 1998, 63, 5129; (b) N. Chatani, T. Fukuyama, F Kakituchi and S. Murai, J. Am. Chem Soc., 1996, 118, 493; (c) E.J. Moore, W.R. Pretzer, T.J. O'Connell, J. Harris, L. LaBounty, L. Chou and S.S. Grimmer, J. Am. Chem. Soc., 1992, 114, 5888

4 (a) W.L. Gladfelter and G.L. Geoffroy, Adus. Organomet. Chem, 1980, 18, 207; (b) L.J. Farrugia, , Advs. Organomet. Chem, 1990, 31, 301; (c) K.H. Whitmire, Advs. Organomet. Chem, 1998, 42,

5 I.D. Salter, Advs. Organomet. Chem, 1989, 29, 249

6 R.D. Adams, T.S. Barnard, Z.Li, W.Wu and J. Yamamoto, Organometallics, 1994, 13, 2357

7 I. Ojima, R.J. Donovan and N. Clos, Organometallics, 1991, 10, 2606

8 J.R. Fox, W.L. Gladfelter and G.L. Geoffroy, I. Tavanaiepour, S. Abdel-Mequid and V.W. Day, Inorg. Chem, 1981, 20,3230

9 L.I. Rubinstein and L.H. Pignolet, Inorg. Chem, 1996, 35, 6755

10 For a recent example see, A.J. Amoroso, M.A. Beswick, C.K.Li, J. Lewis, P.R. Raithby and M.C.R DeArellano, J. Organomet. Chem, 1999, 573, 247

11 S.M. Owen, Polyhedron, 1988, 7,253

12 B.E.G. Johnson and J.S. McIndoe, Coord. Chem. Rev, in press

13 P.J. Dyson, B.F.G. Johnson, J.S. McIndoe and P.R.R. Langridge-Smith, Inorg. Chem, $2000,39,2430$

14 G. Critchley, P.J. Dyson, B.E.G. Johnson, J.S. McIndoe, R.K. O'Reilly and P.R.R Langridge-Smith, Organometallics, 1999, 18, 4090

15 W. Henderson, J. S. Mclndoe, B.K. Nicholson and P.J. Dyson. J. Chem. Soce, Dalton Trans, 1998, 519

16 S.A. Hofstadler, R. Bakhtiar and RD. Smith, J. Chem. Educ., 1996, 73, A82

17 W. Henderson and B.K. Nicholson, J. Chem. Soc, Chem. Commun., 1995, 2531 